\title{
Defects of protein production in erythroid cells revealed in a zebrafish Diamond-Blackfan anemia model for mutation in RPS19
}

\author{
Y Zhang ${ }^{1,2}$, J Ear ${ }^{2}$, Z Yang ${ }^{2}, \mathrm{~K}_{\text {Morimoto }}^{2}$, B Zhang ${ }^{1}$ and S Lin ${ }^{\star, 2}$
}

Diamond-Blackfan anemia (DBA) is a rare congenital red cell aplasia that classically presents during early infancy in DBA patients. Approximately, $25 \%$ of patients carry a mutation in the ribosomal protein (RP) S19 gene; mutations in RPS24, RPS17, $R P L 35 A, R P L 11$, and $R P L 5$ have been reported. How ribosome protein deficiency causes defects specifically to red blood cells in DBA has not been well elucidated. To genetically model the predominant ribosome defect in DBA, we generated an rps19 null mutant through the use of TALEN-mediated gene targeting in zebrafish. Molecular characterization of this mutant line demonstrated that rps19 deficiency reproduced the erythroid defects of DBA, including a lack of mature red blood cells and p53 activation. Notably, we found that rps19 mutants' production of globin proteins was significantly inhibited; however, globin transcript level was either increased or unaffected in rps19 mutant embryos. This dissociation of RNA/protein levels of globin genes was confirmed in another zebrafish DBA model with defects in rp/11. Using transgenic zebrafish with specific expression of mCherry in erythroid cells, we showed that protein production in erythroid cells was decreased when either rps19 or rp/11 was mutated. L-Leucine treatment alleviated the defects of protein production in erythroid cells and partially rescued the anemic phenotype in both rps19 and rp/11 mutants. Analysis of this model suggests that the decreased protein production in erythroid cells likely contributes to the blood-specific phenotype of DBA. Furthermore, the newly generated $r p s 19$ zebrafish mutant should serve as a useful animal model to study DBA. Our in vivo findings may provide clues for the future therapy strategy for DBA. Cell Death and Disease (2014) 5, e1352; doi:10.1038/cddis.2014.318; published online 24 July 2014

Diamond-Blackfan anemia (DBA; OMIM 205900) is a rare congenital bone marrow failure syndrome characterized by red cell aplasia and reduced or absent erythroid precursors. Most patients are diagnosed with anemia during infancy or in the neonatal period. ${ }^{1}$ Additional physical anomalies are present in $30-40 \%$ of DBA patients and include thumb, upper limb, craniofacial, heart and kidney malformations, and short stature..$^{2,3}$ One current treatment option for DBA is bone marrow transplantation; however, this procedure is limited by shortage of appropriate marrow donors and is associated with the risk of significant complications. ${ }^{4,5}$ Therefore, development of drug-based therapeutics for DBA is much needed and desirable.

DBA is a disease associated with ribosome protein mutations. The most common mutations are found in RPS19 that occurs in $\sim 25 \%$ of DBA patients. Frameshift, splicing sites, intragenic deletions, nonsense, as well as missense mutations have all been identified. ${ }^{2}$ Genetic studies have also identified mutations in other ribosomal proteins, including RPS7 in 1\% of patients and RPL11 and RPS26 in $6.5 \% .^{6-12}$ These findings establish DBA as a disorder of ribosomal biogenesis and/or function.

Knockdown of individual ribosomal proteins in human cell lines interferes with pre-rRNA processing and maturation of the large and small ribosome subunits. ${ }^{13-16}$ Presumptively impaired ribosome biogenesis should affect all cell types and understanding why ribosomal protein mutations result in red blood cell-specific defects in DBA remains an interesting but challenging question. It is generally believed that the red blood cell defect is attributed to the activation of p53 in the erythroid lineage caused by ribosomal deficiency. Activation of $p 53$ in DBA is well established but its role in erythropoiesis is controversial. A study by Dutt et al. ${ }^{17}$ reported the selective accumulation of $P 53$ in the erythroid lineage in human hematopoietic progenitor cells after knocking down RPS14 or RPS19 by shRNAs. Inhibition of p53 rescued impaired erythropoiesis in both mouse and zebrafish models of DBA. ${ }^{18,19}$ Recently, p53-independent pathways are beginning to be revealed and demonstrated to be partially responsible for defective erythropoiesis. ${ }^{20,21}$

Another hypothesis to the red blood cell-specific defects in ribosomal protein mutations is due to a shortage of globin proteins. Because blood cells have such a high demand for globin protein, a high rate of protein translation is required; any defects in ribosomal function and/or biogenesis would, therefore, be more severe in blood cells compared to other cell types. Blood cells also have a greater demand for overall protein synthesis due to their highly proliferative and

\footnotetext{
${ }^{1}$ Key Laboratory of Cell Proliferation and Differentiation of Ministry of Education, College of Life Sciences, Peking University, Beijing, People's Republic of China and ${ }^{2}$ Department of Molecular, Cell \& Developmental Biology, University of California Los Angeles, Los Angeles, CA, USA

${ }^{*}$ Corresponding author: S Lin, Department of Molecular, Cell \& Developmental Biology, University of California Los Angeles, Los Angeles, CA 90095, USA. Tel: +01 310267 4970; Fax: +01310 267 4971; E-mail: shuolin@ucla.edu

Abbreviations: DBA, Diamond-Blackfan anemia; RP, ribosomal protein; TALEN, transcription activator-like effector nuclease; dpf, days post fertilization; HSC, hematopoietic stem cell; hbbe1, hemoglobin beta e1; hbbe3, hemoglobin beta e3

Received 21.2.14; revised 12.5.14; accepted 14.5.14; Edited by E Baehrecke
} 
differentiating nature. Protein synthesis rate was reported to be reduced in lymphocytes from DBA patients irrespective of RPS19 mutations. ${ }^{22}$ Studies of mouse erythroblasts with ribosomal protein haploinsufficiency revealed impaired translation of specific transcripts essential for erythropoiesis involving internal ribosomal entry site (IRES). ${ }^{23}$ In addition, human K562 cells were shown to express alternatively spliced isoforms of FLVCR1 transcripts, which were affected by expressing shRNA against RPS19. ${ }^{24}$ Dysfunction of FLVCR1 as a heme exporter in erythroblasts ${ }^{25}$ might also play a role in the erythroid defects of DBA in addition to defective translation rate. Furthermore, decreased expression of the key erythroid signaling protein KIT was detected in fetal liver cells of a RPS19-deficient mouse, which may contribute to the erythroid failure in DBA. ${ }^{26}$ Although much has been discovered utilizing various models of ribosome deficiency, the exact mechanism of erythroid failure in DBA remains largely unknown. More in vivo experiments in various models are needed to elucidate the pathogenesis of DBA.

To gain a better understanding of the mechanism leading to the erythroid-specific defects in DBA, we generated two independent lines of rps19 mutants using transcription activator-like effector nuclease (TALEN)-mediated gene targeting in zebrafish. ${ }^{27}$ Genetic knockout of rps 19 resulted in the characteristic erythroid defects similar to DBA including a lack of mature red blood cells and p53 activation. Interestingly, we observed that rps19 mutants had significantly reduced production of globin proteins accompanied by either increased or unaffected level of mRNA transcripts. Similarly, this phenomenon was observed in rp/11 mutant zebrafish. Furthermore, we observed that protein production in erythroid cells was decreased with either a mutation in rps 19 or rpl11. Treatment with L-leucine improved the defect of protein production and partially rescued the anemic phenotype in both rps 19 and rp/11 mutants. These findings suggest that decreased protein production in red blood cells is likely a key contributing factor to erythroid-specific defect in DBA.

\section{Results}

Generation of rps19 mutant with TALENs. Rps 19 was the first mutated gene identified in human DBA patients. ${ }^{2}$ Zebrafish have a single copy of the rps19 gene that shares over $88 \%$ amino acid sequence identity with the human RPS19 gene. To generate a zebrafish DBA model carrying rps19 mutation, TALEN target sites were designed at the boundary of the second intron and the third exon of the rps19 gene (Figure 1a). The left and right TALENs were co-injected in zebrafish embryos at the one-cell stage to induce insertions or deletions (indels) into the rps19 gene (Figure 1b). This resulted in premature stop codons in the mRNA (Figure 1c). Two independent stable germline mutations of rps19 were established (Figures $1 \mathrm{~b}$ and $\mathrm{c}$ ). Both rps19 mutant lines displayed a similar phenotype and we chose the first mutant line (noted as 'Mu1' in Figure 1c) for further investigation. Expression analysis of rps 19 mRNA showed $>90 \%$ reduction in the rps 19 homozygous mutants (Figure 1d) likely due to nonsense-mediated decay of mRNA. Because mutations of RPS19 in DBA patients include allele
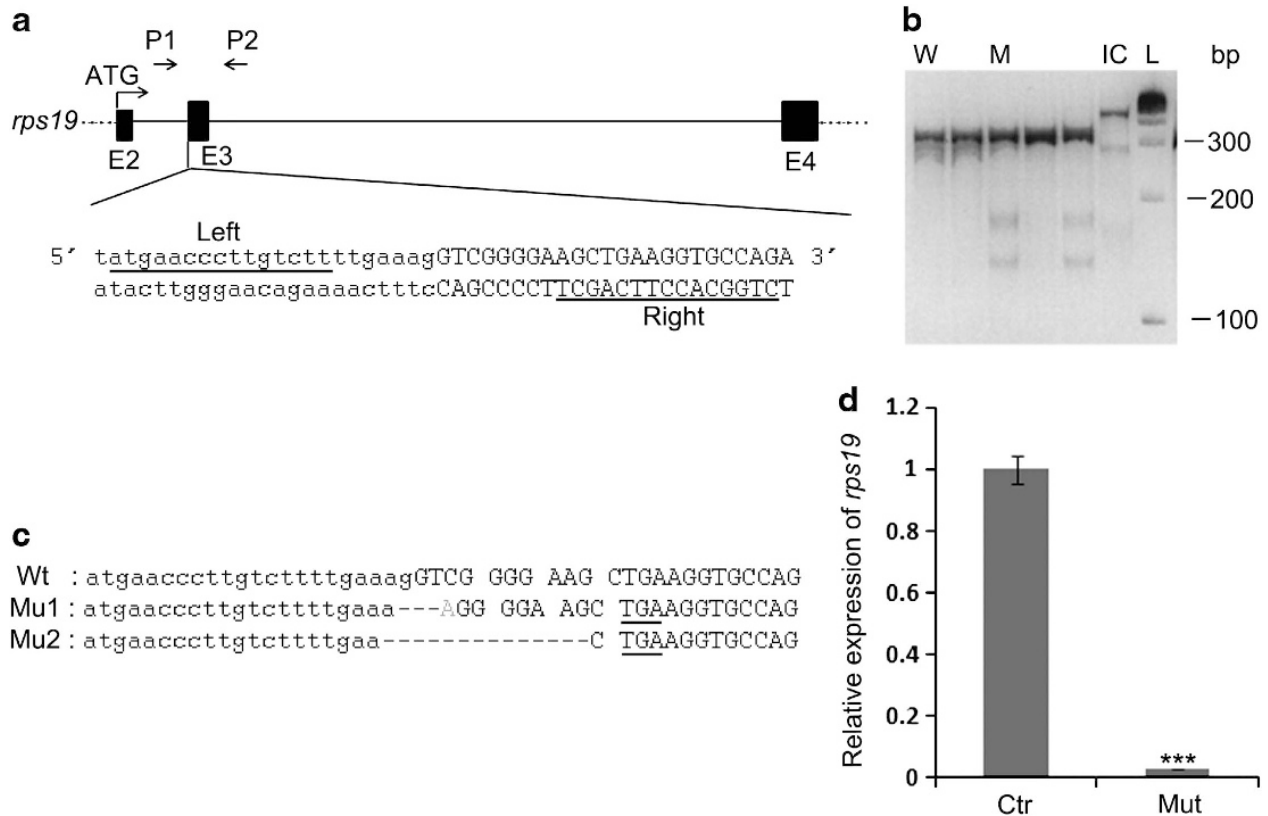

Figure 1 The rps19 gene in zebrafish is targeted using TALENs. (a) Partial structure and sequence of the zebrafish rps19 gene. The binding sites of the left and right TALENs were underlined. The intron sequence was shown in lowercase and the exon in uppercase. P1 and P2 showed the primer sites for amplification. E2, E3, and E4 represent the second, third, and fourth exon of the rps19 gene, respectively. (b) Identification of mutations by enzyme digestion. PCR products ( $\sim 305 \mathrm{bp})$ were processed with an enzyme that can recognize and cut at mismatch region. The presence of lower bands ( $\sim 135 \mathrm{bp}$ and $\sim 170 \mathrm{bp}$ ) suggested a mutation. (c) Genomic sequences of the rps19 mutations. Deletions and insertions were shown in dashes and red letters, respectively. The premature stop codons produced in the two independent mutations were underlined. (d) Real-time PCR result showed rps 19 gene expression was reduced by more than $99 \%$ in mutant $1 .{ }^{* \star} P<0.001 ;$ Student's $t$-test). W, WT control; M, mutant; IC, internal control; L, DNA Ladder; bp, base pair 
deletion, missense mutations, premature stop codons, and internal deletions, we reason that the rps 19 mutant zebrafish would mimic the premature stop codon mutations found in DBA patients.

Phenotypic analysis of rps19 mutants. Rps19 homozygous mutants exhibited a similar phenotype as that of rps 19 morphants. ${ }^{28}$ At $24 \mathrm{~h}$ post fertilization (hpf), rps19 homozygous mutants showed abnormal development of midbrain-hindbrain boundary (Figure 2a). A curved tail was observed in the mutant embryos at $48 \mathrm{hpf}$. Compared with siblings, rps19 mutants exhibited smaller heads and smaller eyes at 3 days post fertilization (dpf). The mutants developed edema (Figure $2 \mathrm{a}$ ) and died $\sim 5 \mathrm{dpf}$.

To determine if our rps 19 mutants displayed a DBA-like hematopoietic phenotype, we investigated the expression of multiple primitive and definitive hematopoietic markers using RNA whole-mount in situ hybridization. The initial a

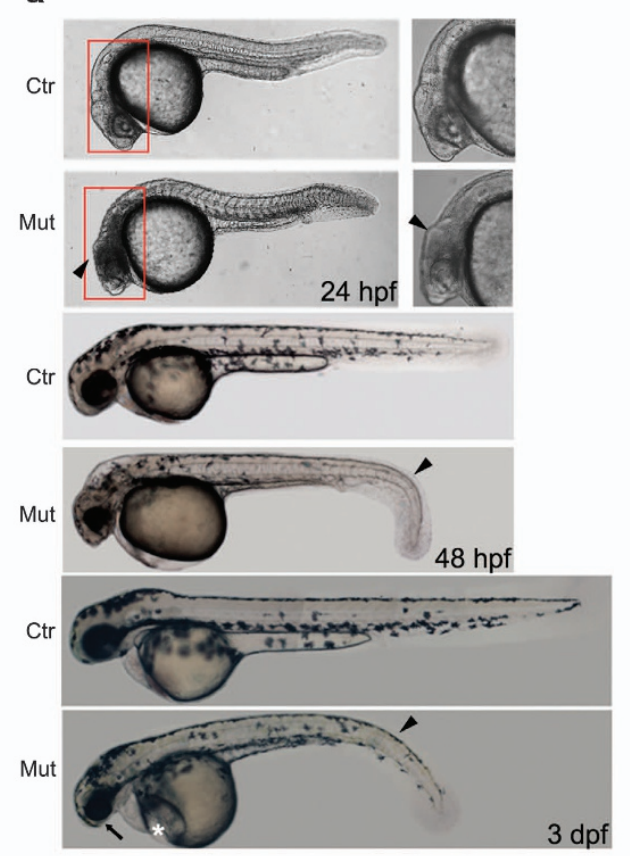

b

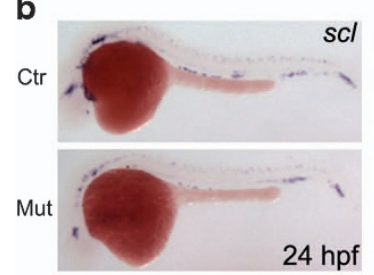

Ctr

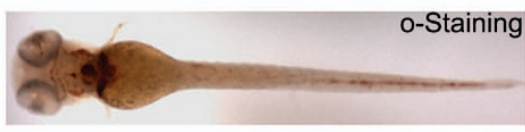

Mut

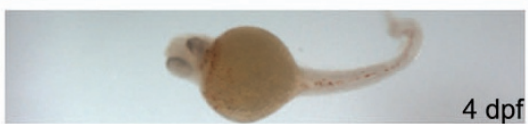

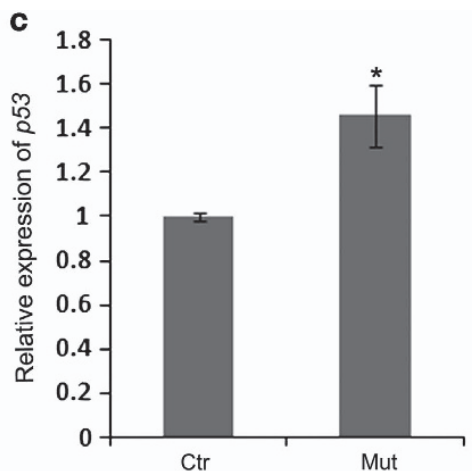

d

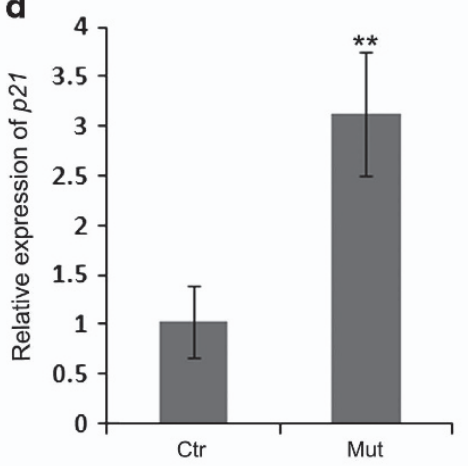

c-myb

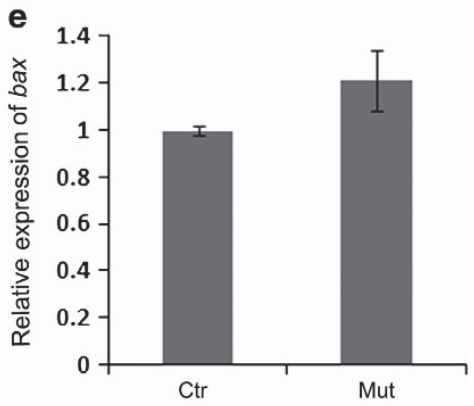

Figure 2 Phenotypic analysis of rps 19 mutants. (a) Pictures of embryos at different stages showed the phenotype of rps 19 homozygous mutants. At $24 \mathrm{~h}$ post fertilization (hpf), midbrain-hindbrain boundary (MHB) poorly developed in the homozygous mutant embryos (black arrowheads). Boxed areas on the left side were magnified on the right side. Curved tail was observed in homozygous mutant embryos at $48 \mathrm{hpf}$ (black arrowhead). Black arrow showed the smaller head and smaller eyes in homozygous mutant embryos at 3 days post fertilization (dpf). Compared with siblings, the mutant embryos developed edema (white star). The black arrowhead indicated the curved tail in mutant embryos. (b) Analysis of hematopoietic defects in rps19 homozygous mutant embryos. The primitive hematopoietic stem cells (HSCs), marked by scl were not affected or slightly less in the mutant embryos at $24 \mathrm{hpf}$. Both the expression of c-myb in definitive HSC at $3 \mathrm{dpf}$ and the hemoglobin stained by o-dianisidine staining at $4 \mathrm{dpf}$ were almost disappeared in the mutant embryos. The number of lymphoid cells, marked by rag1, was obviously decreased in the mutant at $4 \mathrm{dpf}$. All the embryos in panel a and those for $s c l$ and $c$-myb probes in panel $\mathbf{b}$ are lateral view with anterior to the left. The embryos for 0 -dianisidine staining (o-staining) are ventral view with anterior to the left. And the embryos for rag1 probe are dorsal view with anterior to the left. (c) Real-time PCR results showed the expression of p53 was upregulated in rps19 homozygous mutant embryos. (d and e) Some of the downstream targets of $p 53$ were also upregulated in mutant embryos, including $p 21$, which is responsible for the induction of cell-cycle arrest and bax, which is a mediator of apoptosis. RNA was pooled from 20 embryos at $3 \mathrm{dpf}$. The results are representative of three independent experiments. ${ }^{*} P<0.05 ;{ }^{* \star} P<0.01$; Student's t-test 
specification of the primitive hematopoietic cells located in the intermediate cell mass of zebrafish as marked by $\mathrm{scl}$ expression was not highly affected in rps 19 homozygous mutants at $24 \mathrm{hpf}$ (Figure 2b). However, compared to siblings, expression of the definitive hematopoietic stem cells (HSCs) marker c-myb was nearly absent in rps 19 mutants at $3 \mathrm{dpf}$. o-Dianisidine staining revealed that rps19 mutants had greatly decreased hemoglobin levels at $4 \mathrm{dpf}$ (Figure 2b). This indicated that the number of red blood cells was probably reduced in rps19 mutants. The reduction of red blood cells may be caused by a decreased cell survival and/or production of definitive HSCs. This is supported by the observation that lymphoid $\mathrm{T}$ cells in the thymus, marked by rag1 expression, were also nearly lacking in rps 19 mutants at $4 \mathrm{dpf}$ (Figure 2b).

Upregulation of the $p 53$ signaling pathway was reported in the majority of DBA patients, DBA cell culture models, and animal models. As expected, rps19 deficiency in our mutants led to activation of the p53 signaling pathway (Figure 2c), including $p 53$ and its target genes such as the cell-cycle arrest gene, p21 (Figure 2d), and the apoptotic gene, bax (Figure $2 e$ ). In addition, similar with the results previously found in rps29 mutants and other ribosomal protein-deficient models, ${ }^{29,30}$ knockdown of $p 53$ expression could partially rescue mutant phenotype observed in rps19 homozygous mutants, including the morphological phenotype. Anemic phenotype observed in rps19 mutants was also partially improved by downregulation of $p 53$ (Supplementary Figure S1). In conclusion, the rps19 mutants we generated with TALENs exhibited the characteristic phenotype of DBA patients and should be useful as a model to study pathogenesis of this genetic disease.

RNA and protein levels of globin genes are dissociated in rps19 mutants. The primary phenotype of DBA is anemia. In the course of characterizing embryonic hemoglobin gene expression in rps 19 mutants we noted that mRNA for hemoglobin beta e1 (hbbe1) was not reduced whereas hemoglobin gene hbbe3 mRNA was increased by $>50$-fold (Figures $3 a$ and $b$ ). This was somewhat unexpected given that rps19 mutants lacked globin proteins as revealed by o-dianisidine staining. To reconcile this issue, we obtained antibodies specifically recognizing hbbe1 or hbbe3 proteins and quantified globin protein levels by western blot analysis. As expected, we observed significant reduction of protein for both hbbe1 and hbbe3 (Figure 3c), suggesting that there is dissociation of RNA and protein levels for globin gene expression in rps 19 mutants. As a control, we analyzed both the mRNA and protein levels for neutrophil marker mpx. We detected $\sim 50 \%$ less mpx mRNA in rps 19 mutants, compared with control embryos (Figure 3d). However, no reduction in mpx protein was detected in rps19 mutants (Figure 3e). This indicates that the dissociation of RNA and protein production is not ubiquitous but at least partially erythroid cell-specific or globin gene-specific effect.

Protein production in erythroid cells is decreased in rps19 mutants. Globin is the most abundant protein in red blood cells. The dissociation of globin genes' RNA and protein production could result from the deficient protein production in erythroid cells. Alternatively, the dissociation can be due to globin gene-specific pathogenesis. In order to address these questions, we generated transgenic zebrafish specifically labeling red blood cells with mCherry. As shown in Figure 4a, zebrafish erythroid-specific regulatory region locus control region (LCR) as well as a portion of $\alpha / \beta_{\mathrm{a} 2}$ bidirectional proximal promoter ${ }^{31}$ was cloned into mCherry reporter constructs. Because the dissociation of RNA and protein production was more significant for hbbe3 than hbbe1, we included the $5^{\prime}$ untranslated region (UTR) or $3^{\prime}$ UTR of hbbe3 in these constructs (Figure $4 a$ ). We observed robust erythroid-specific expression of mCherry in these transgenic embryos, especially in mCherry control line (Supplementary Figure S2). We examined if the globin gene structure has any effects on mCherry expression at the mRNA level and protein level in rps19 mutants. Real-time PCR results showed equal to more mCherry mRNA in mCherry-positive rps19 mutant embryos than the control embryos at $3 \mathrm{dpf}$ (Figure 4b). However, less mCherry protein, measured through fluorescent intensity, was detected in rps19 mutants compared to siblings in all three transgenic lines (Figures 4c and $d$ ). Interestingly, this RNA/protein production dissociation of mCherry was more severe in mCherry control line. Therefore, we observed the dissociation of RNA/protein levels with erythroid-specific mCherry expression regardless of gene structure of hbbe3. This indicates that protein production in erythroid cells was inhibited in rps19 mutants.

RNA and protein levels of globin genes are also dissociated in rpl11 mutants. Previously, a rp/11 mutant identified with a retrovirus-mediated mutation screening library, ${ }^{32}$ has been established as a zebrafish DBA model for its anemic phenotype, p53 upregulation, and metabolic defects. $^{30}$ To determine if the RNA/protein production dissociation for globin gene expression was specific to rps19 mutant or general to DBA-associated ribosomal protein deficiency, we analyzed mRNA expression and protein level in zebrafish rp/11 mutants. Real-time PCR results showed slightly increased expression of hbbe1 mRNA in rp/11 mutant (Figure 5a), while expression of hbbe3 mRNA was increased by $\sim 50$-folds in rp/11 mutants compared to control embryos (Figure 5b). Furthermore, slightly less mpx mRNA was detected in rpl11 mutants at $3 \mathrm{dpf}$ (Figure 5d). Whole-mount in situ hybridization analysis confirmed the real-time PCR as equal to more hbbe1 mRNA was observed in rp/11 mutants compared with siblings at $3 \mathrm{dpf}$ (Supplementary Figure S3a). Expression of hbbe3 mRNA was significantly increased in rpl11 mutant (Supplementary Figure S3b); mpx exhibited similar expression levels between the siblings and rp/11 mutants (Supplementary Figure S3c). As we discovered in rps19 mutants, less hbbe1 and hbbe3 proteins were also shown in rp/11 mutants by western blot analysis (Figure $5 \mathrm{c}$ ), whereas protein level of $m p x$ was comparable between control and rpl11 mutants (Figure 5e). These findings demonstrated that the RNA/protein production dissociation of globin genes without concurrent dissociation seen in the neutrophil cells is likely a common phenomenon shared between multiple ribosome mutations found in DBA. 
a
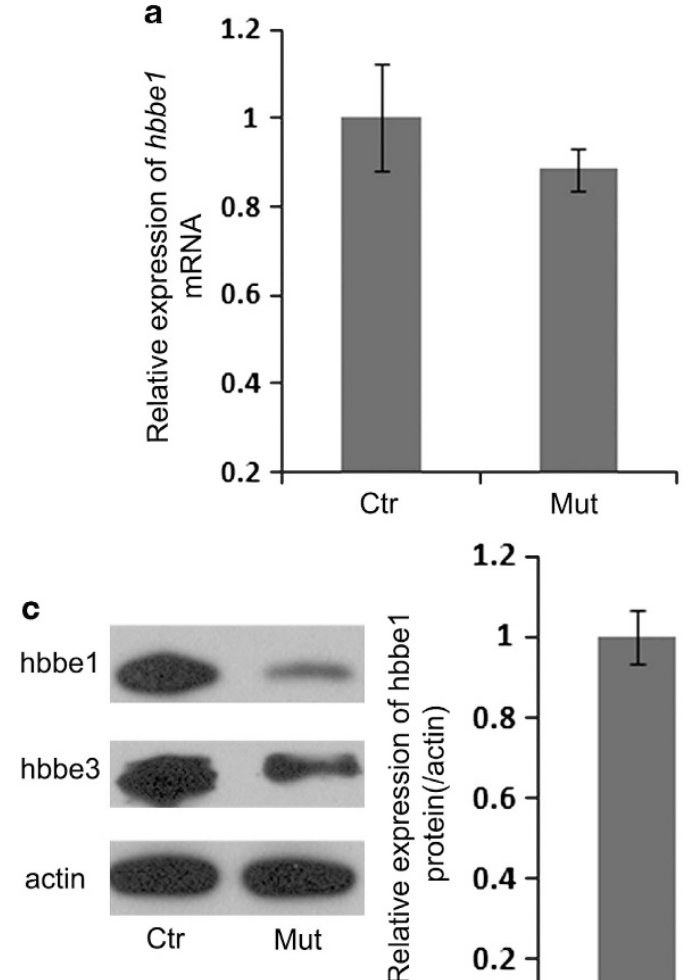

d

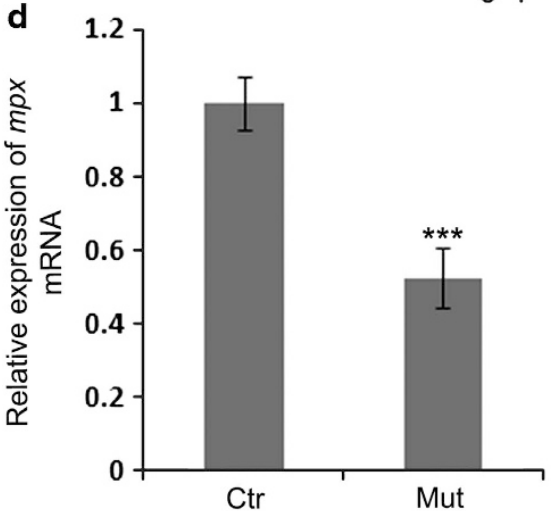

b

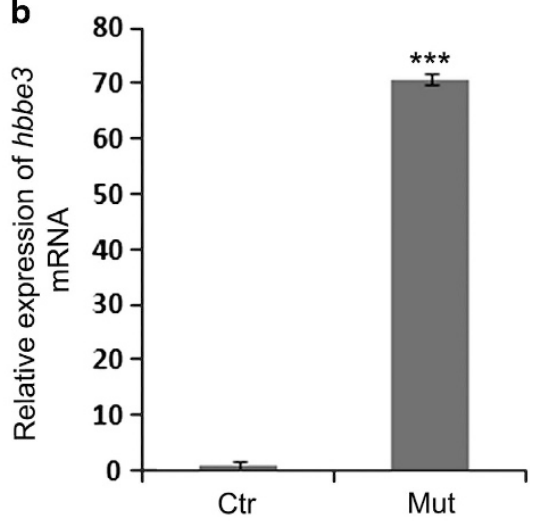

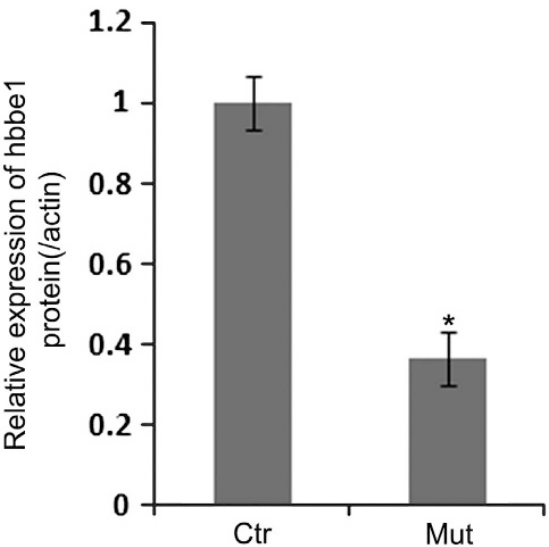
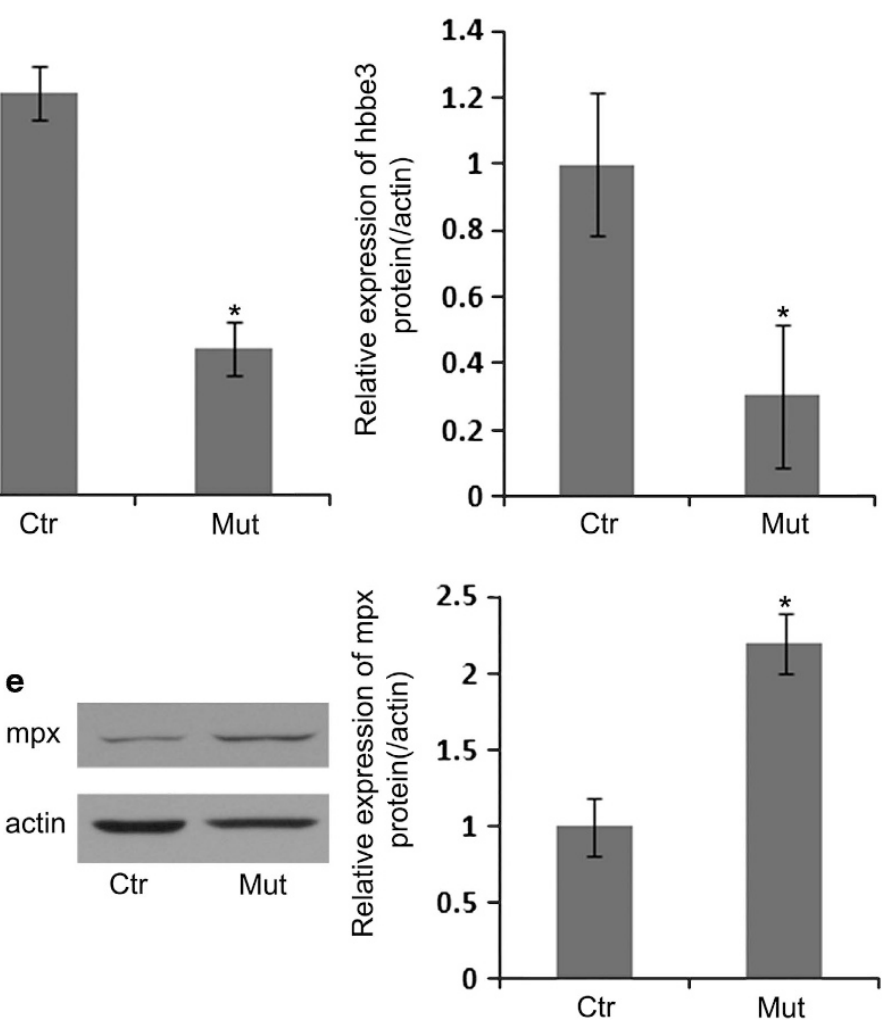

Figure 3 RNA and protein levels of globin genes are dissociated in rps 19 mutants. (a) mRNA expression of globin gene hemoglobin beta e1 (hbbe1) was not affected or slightly decreased in rps 19 mutant embryos at $3 \mathrm{dpf}$ by real-time PCR analysis. (b) Real-time PCR results showed $\sim 70$-fold more hbbe3 mRNA was accumulated in rps 19 mutant embryos than control embryos. (c) Western blot results indicated protein levels of both $h b b e 1$ and $h b b e 3$ were dramatically decreased in rps 19 mutant embryos at $3 \mathrm{dpf}$. Histograms represented quantification of western blots. (d) Decreased mpx mRNA was detected in rps19 mutants compared with control embryos. (e) Equal to more mpx protein was produced in rps 19 mutants than control embryos at $3 \mathrm{dpf}$. Histograms represented quantification of western blots. Actin acts as a loading control. The results are representative for three independent experiments. ${ }^{*} P<0.05 ;{ }^{* \star *} P<0.001$; Student's $t$-test

Protein production in erythroid cells is also decreased in rpl11 mutants. To assess protein production in erythroid cells in rpl11 mutants, we bred the transgenic lines into the rpl11 mutant line. As expected, approximately two- to fivefold more mCherry mRNA was detected by real-time PCR in mCherry-positive rp/11 mutant embryos compared with siblings at $3 \mathrm{dpf}$ (Figure 6a). However, less mCherry protein was produced in rp/11 mutants (Figures 6b and c). This suggests that protein production in erythroid cells was also inhibited in rp/11 mutants. Similar with rps 19 mutants, the dissociation of RNA and protein levels of mCherry was more obvious in rp/11 mutants of mCherry control transgenic line.

To exclude the possibility that general protein translational defect led to erythroid protein production deficit, we examined the translational rates in both models. Cells from wild-type control embryos and rps 19 mutants were respectively starved by culturing in methionine and cysteine-free medium. Following starvation, ${ }^{35} \mathrm{~S}$-labeled methionine and cysteine were incorporated into those cells and the radioactivity was measured. As shown in Supplementary Figure S4a, the global translation rate was reduced only $\sim 40 \%$ in rps 19 
mutants compared to the control group, which was much less significant than the reduction of erythroid-specific mCherry protein levels in rps19 transgenic mutants ( 70-85\%).
We performed the same experiments with rp/11 mutants. Results showed that general translation rate was comparable between control embryos and rp/11 mutants

a
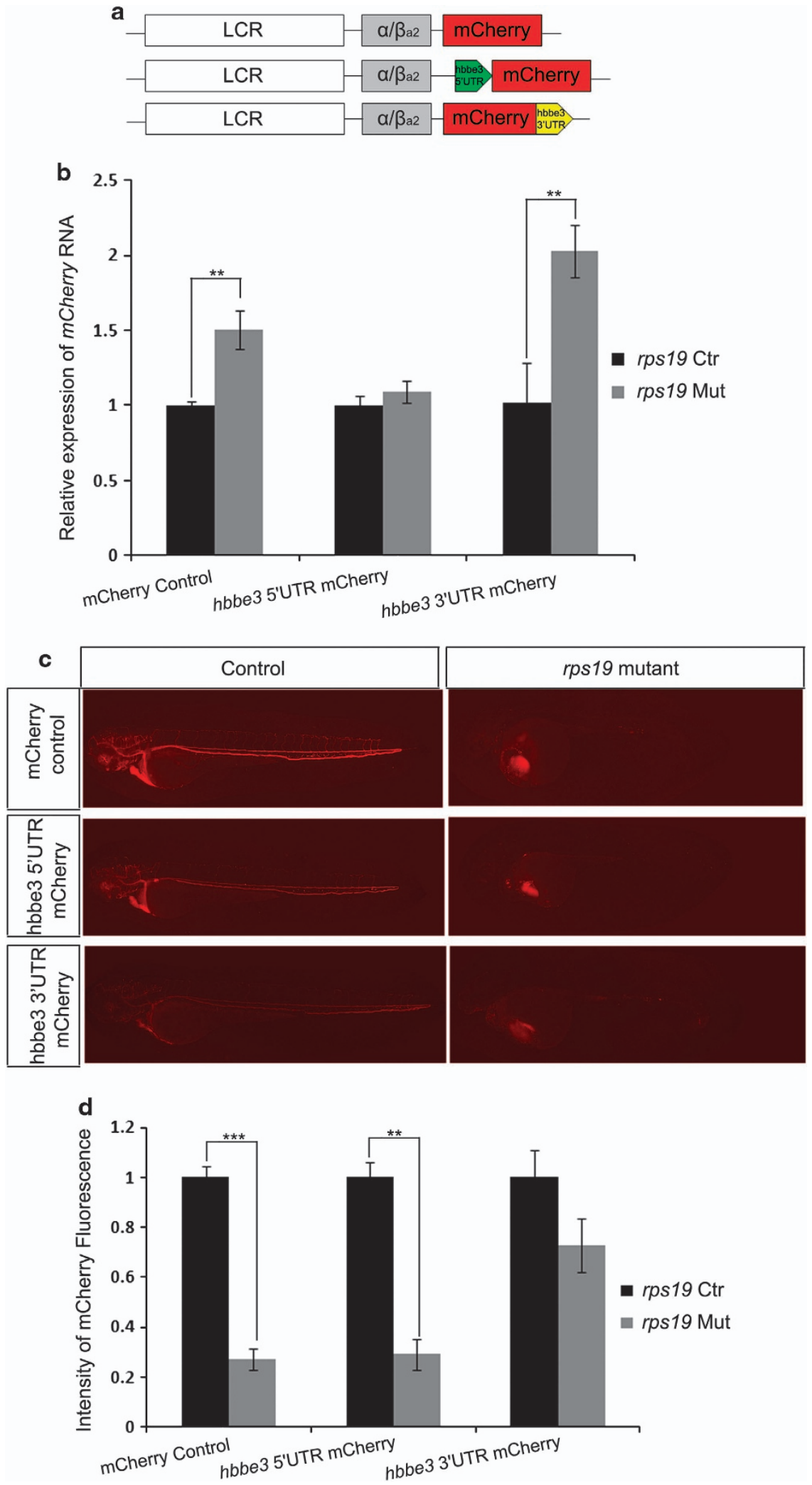
(Supplementary Figure S4b). These findings suggest that the erythroid protein production defect we observed in both rps19 mutants and rp/11 mutants was not simply contributed by general translational defect.
Meanwhile, expression of gata1, which is essential for differentiation of erythroid progenitor cells, was unaffected or slightly reduced in both rps 19 mutants and $r p / 11$ mutants, with more gata 1 mRNA was detected in both mutant models at
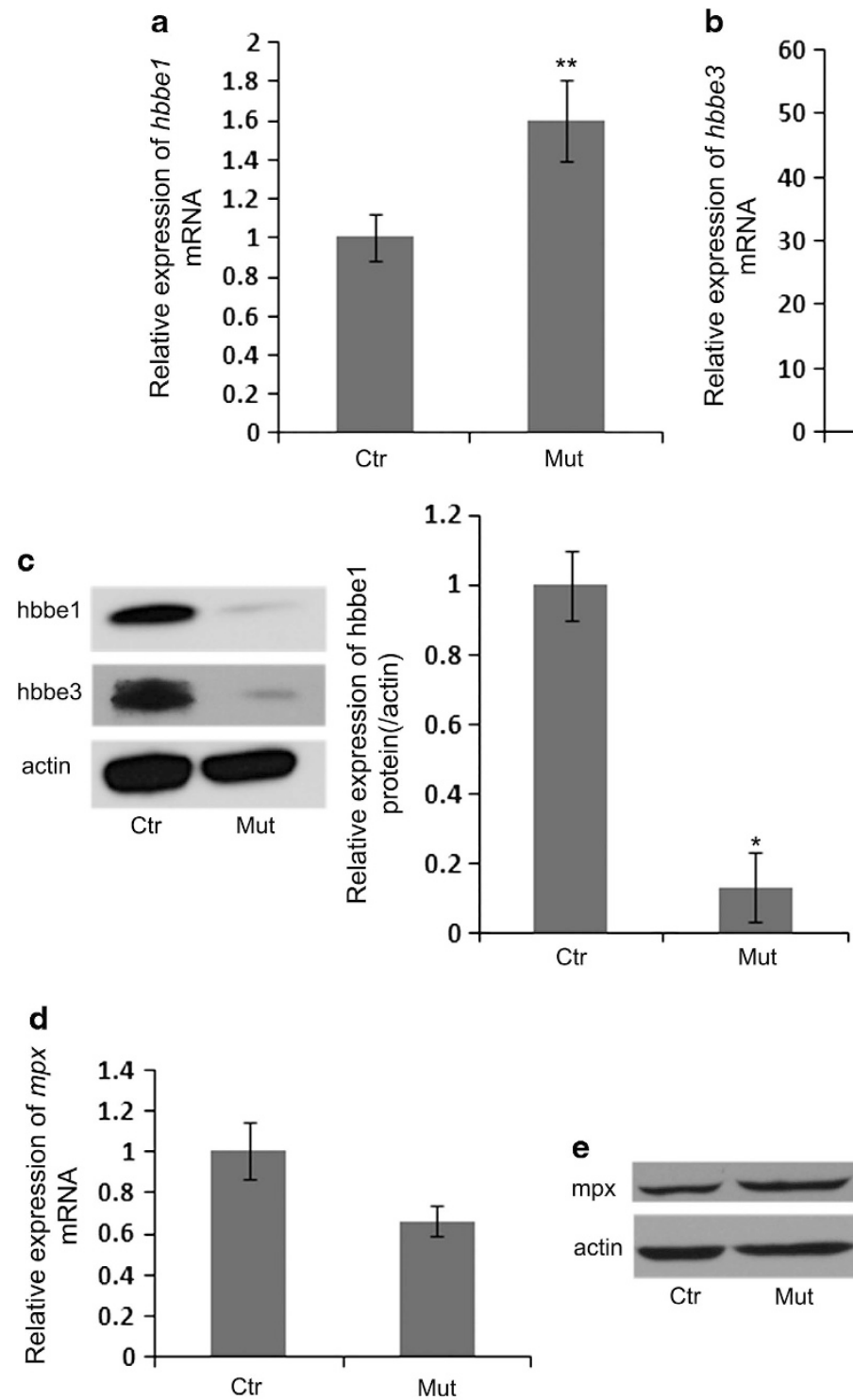

b
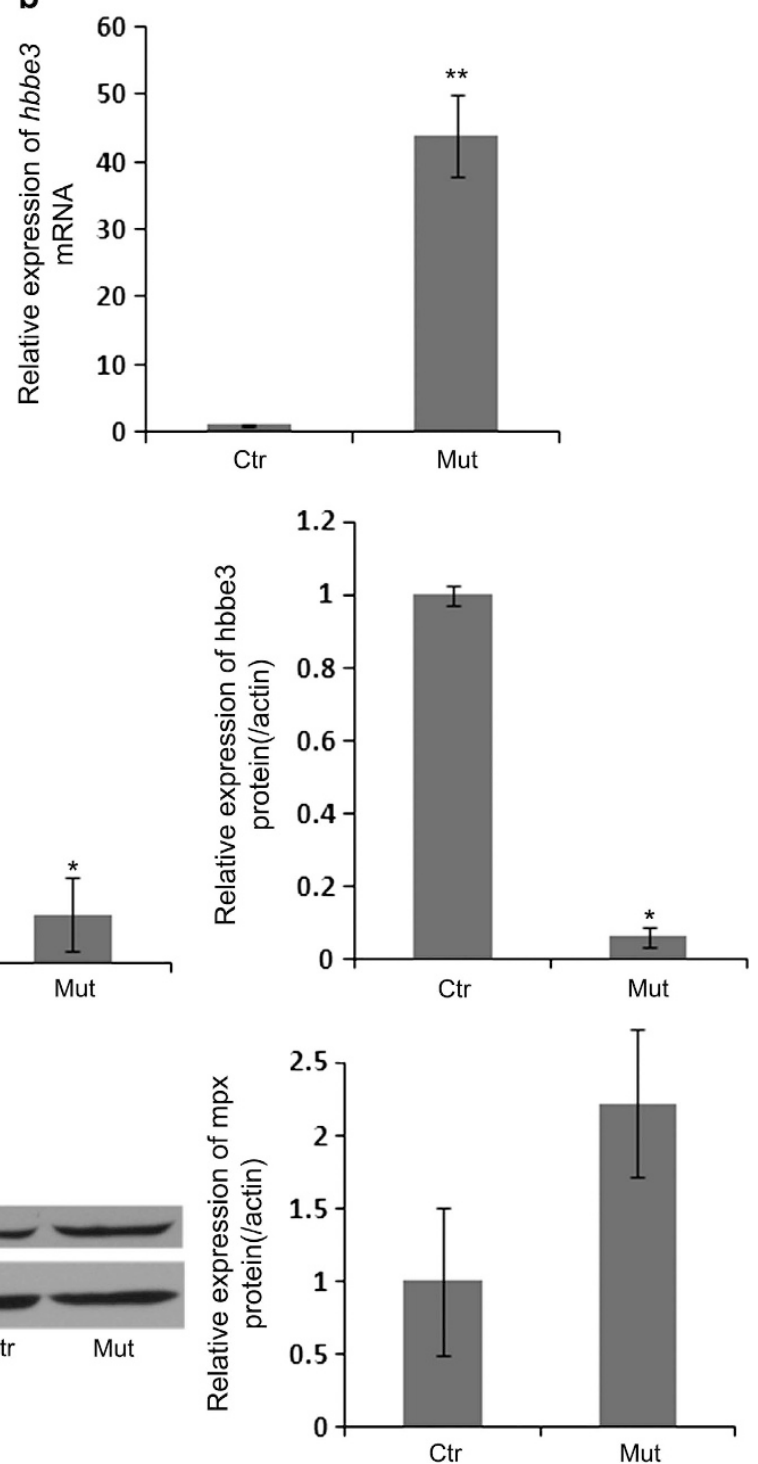

Figure 5 RNA and protein levels of globin genes are also dissociated in rp/11 mutants. (a) mRNA expression of $h b b e 1$ was increased to 1.5 -fold in rpl11 mutant embryos at $3 \mathrm{dpf}$ by real-time PCR analysis. (b) Real-time PCR results showed $\sim 50$-fold more hbbe3 mRNA was accumulated in rpl11 mutant embryos compared to control embryos. (c) Western blot results indicated dramatic decrease of hbbe1 and hbbe3 protein levels in rpl11 mutants at 3 dpf. Histograms represented quantification of western blots. (d) Slightly decreased mpx mRNA was detected in rpl11 mutants compared with control embryos. RNA was pooled from 10 embryos. (e) Mpx protein was detected by western blot. Expression of mpx protein was comparable between rpl11 mutant embryos and siblings, or more mpx protein was detected in rp/11 mutant embryos than siblings. Histograms represented quantification of western blots. Actin acts as a loading control. The results are representative for three independent experiments. ${ }^{*} P<0.05$; ${ }^{* *} P<0.01$; Student's t-test

Figure 4 Protein production in erythroid cells is decreased in rps19 mutants. (a) Representation of the constructs for generating transgenic zebrafish lines. Zebrafish erythroid-specific regulatory region - locus control region (LCR) as well as a portion of $\alpha / \beta_{\mathrm{a} 2}$ bidirectional proximal promoter was cloned into mCherry reporter constructs. $5^{\prime}$ UTR or $3^{\prime}$ UTR of hbbe3 was also included into one of the constructs. (b) Real-time PCR results showed more or the same amount of mCherry RNA in mCherry-positive rps19 mutant embryos compared with control group at $3 \mathrm{dpf}$. (c) Representative images of mCherry-positive embryos of different transgenic lines at $3 \mathrm{dpf}$. Less mCherry expression was observed in rps19 mutants than control embryos in all three transgenic lines. Lateral view shown with anterior to the left. (d) Quantification of intensity of mCherry fluorescence in the embryos shown in c. Intensity of mCherry fluorescence shown in rps 19 mutant mCherry control transgenic embryos was only $27 \%$ of the siblings. Approximately, $30 \%$ of mCherry fluorescence was exhibited by rps 19 mutant hbbe $35^{\prime}$ UTR mCherry transgenic embryos, compared with the control embryos. The results are representative for three independent experiments. ${ }^{\star * \star} P<0.001 ;{ }^{* \star} P<0.01$; Student's $t$-test 

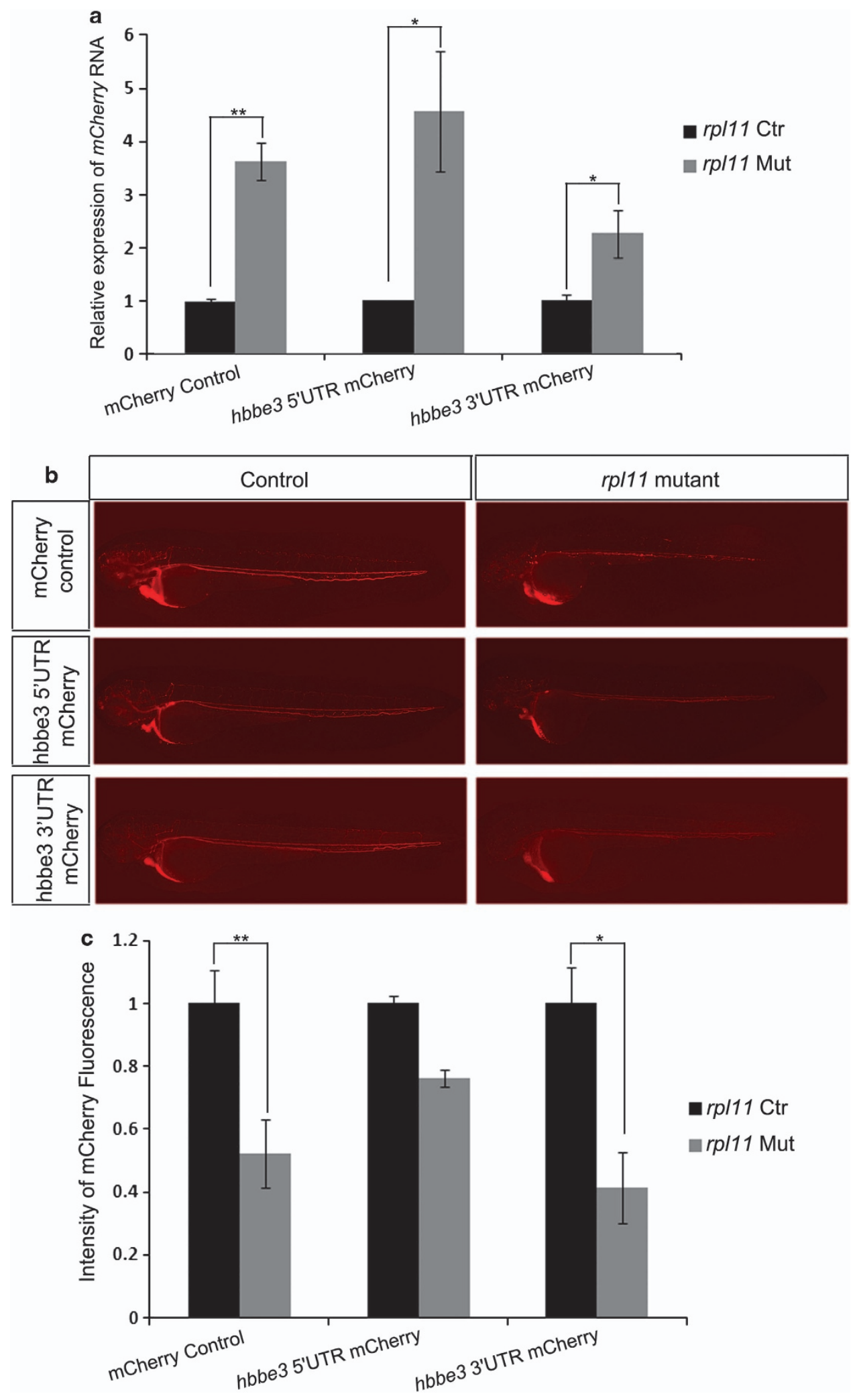

Figure 6 Protein production in erythroid cells is also decreased in rpl11 mutants. (a) Real-time PCR results showed more than twofolds of $m C h e r r y$ RNA accumulated in mCherry-positive rp/11 mutant embryos compared with control groups at $3 \mathrm{dpf}$. (b) Representative images of mCherry-positive embryos of different transgenic lines at 3 dpf. Less mCherry expression was observed in rp/11 mutants than control embryos. Lateral view shown with anterior to the left. (c) Quantification of intensity of mCherry fluorescence in the embryos shown in $\mathbf{b}$. Intensity of mCherry fluorescence shown in rpl11 mutant mCherry control transgenic embryos was $\sim 50 \%$ of the siblings. Approximately, $40 \%$ of mCherry fluorescence was exhibited by rpl11 mutant hbbe3 $3^{\prime} \mathrm{UTR}$ mCherry transgenic embryos, compared with the control embryos. The results are representative for three independent experiments. ${ }^{*} P<0.01 ;{ }^{*} P<0.05$; Student's $t$-test 
$3 \mathrm{dpf}$ (Supplementary Figure S5a-d). This indicates the production of erythroid progenitors is probably unaffected. In addition, no significant difference was detected in the percentage of mCherry-positive cells in both rps 19 and rpl11 mutant transgenic embryos from $\mathrm{Tg}$ (LCR: mCherry) at $3 \mathrm{dpf}$ (Supplementary Figure S5e and f), suggesting that the defects of protein production in erythroid cells were not simply due to reduction of erythroid cell numbers.

Furthermore, to exclude any adverse effect of blood circulation on the defects of gene expression in erythroid cells in both ribosomal mutation models, we injected rhodamine fluorescent dye into heart and analyzed the status of circulation at $3 \mathrm{dpf}$. Results showed that circulation was intact in both rps 19 and rp/11 mutants (Supplementary Figure S6). This indicates that deficits of protein production in erythroid cells observed in both ribosomal mutation models were not caused by impaired circulation.

Taken together, for the first time we uncovered an RNA/ protein production dissociation of globin genes in both rps19 and rpl11 mutants. Furthermore, defective protein synthesis in erythroid cells was seen in both rps19 and rp/11 mutants. This indicates that accumulative defects of protein production in erythroid cells probably contribute to the reduction of globin proteins, which leads to the blood-specific defects in DBA patients.

L-leucine treatment alleviates the decreased protein production of globin genes and partially rescues the anemic phenotype in both rps19 and rpl11 mutants. L-Leucine is known to increase protein synthesis in skeletal muscle. ${ }^{33}$ Protein synthesis was increased in DBA-derived cells after treatment with L-leucine. ${ }^{22}$ L-Leucine treatment improved anemia in several animal models with ribosomal protein deficiency. ${ }^{34,35}$ To evaluate the effect of L-leucine treatment on our disease models, we treated both rps 19 and rpl11 mutants with L-leucine. Compared to control, addition of L-leucine improved part of the developmental defects, including edema, in both rps19 and rp/11 mutants (Figures $7 \mathrm{a}$ and $8 \mathrm{a}$ ). To assess if L-leucine treatment plays a role in the protein synthesis defect of globin genes described above, we analyzed expression of hbbe3. Realtime PCR results showed that expression of $h b b e 3$ at mRNA level was comparable between control mutant embryos and mutant embryos raised in L-leucine in both rps19 and rp/11 mutants at $3 \mathrm{dpf}$ (Figures $7 \mathrm{~b}$ and $8 \mathrm{~b}$ ). However, protein level of hbbe3 was significantly increased in mutant embryos a
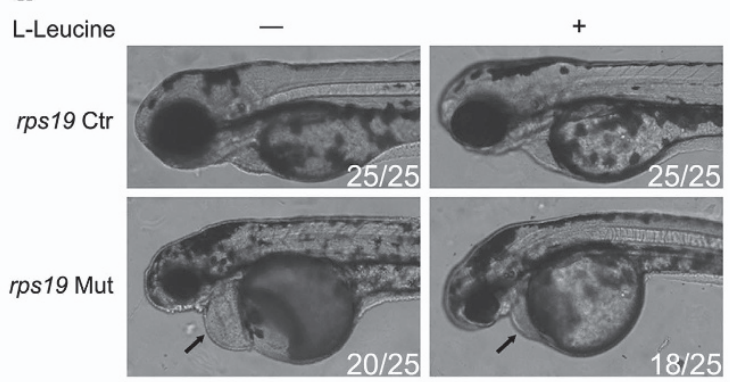

b

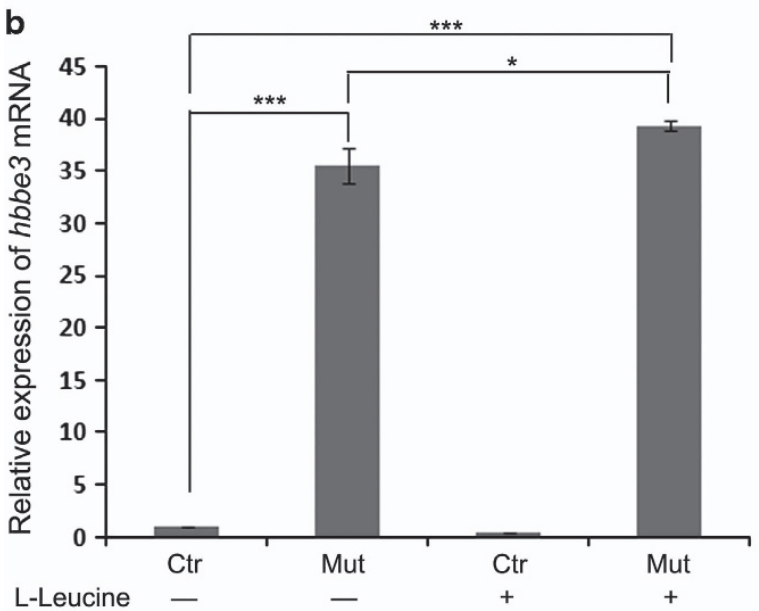

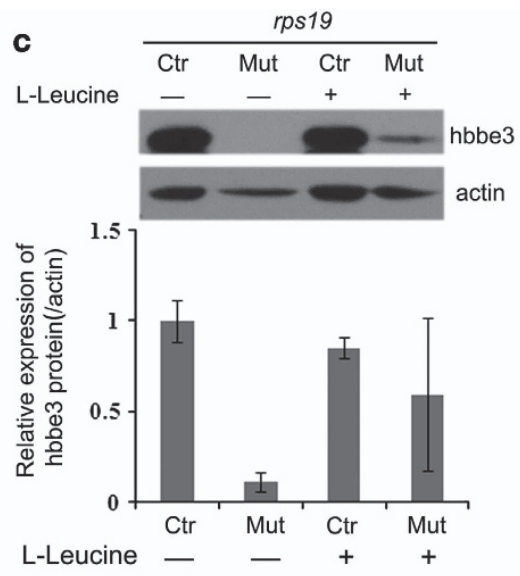

d

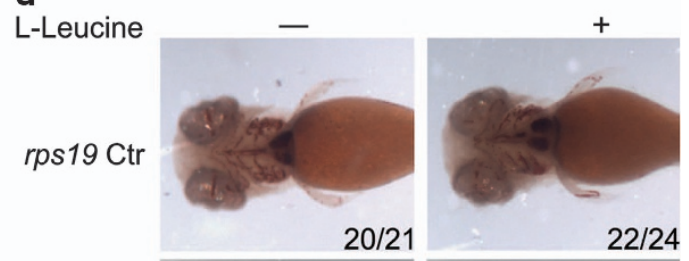

rps19 Mut
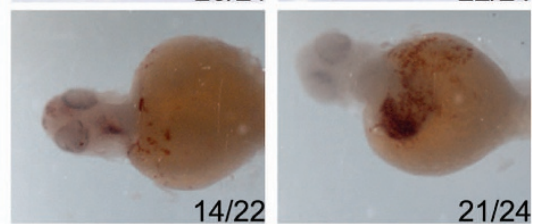

Figure 7 L-Leucine treatment alleviates the protein production defect of globin gene and partially rescues the anemic phenotype in rps 19 mutant embryos. (a) Rps 19 mutant embryos raised in fish water developed severe edema (black arrow) at $3 \mathrm{dpf}$, while the edema was improved after L-leucine treatment in rps 19 mutant embryos (black arrow). L-Leucine had no effect on control embryos. Lateral view with anterior to the left. (b) Real-time PCR results showed that mRNA expression of $h b b e 3$ was slightly increased in rps 19 mutant embryos after addition of L-leucine compared with mutant embryos in fish water at 3 dpf. (c) hbbe3 protein expression was detected by western blot analysis. Expression of hbbe 3 was dramatically increased by treatment of L-leucine in rps 19 mutant embryos at $3 \mathrm{dpf}$. Histograms represented quantification of western blots. Actin acts as a loading control. (d) More hemoglobin staining signals were detected in rps19 mutant embryos treated with L-leucine than the mutant embryos raised in fish water at $4 \mathrm{dpf}$. Ventral view with anterior to the left. The results are representative for two independent experiments. ${ }^{*} P<0.05 ;{ }^{* * *} P<0.001$; Student's $t$-test 
a

L-Leucine

rpl11 Ctr

rpl11 Mut

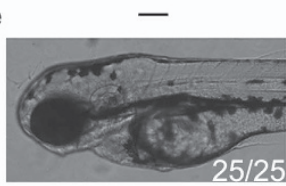

$25 / 25$
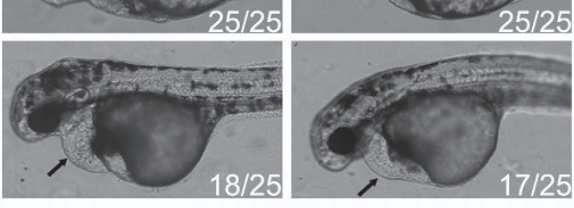

\section{b}

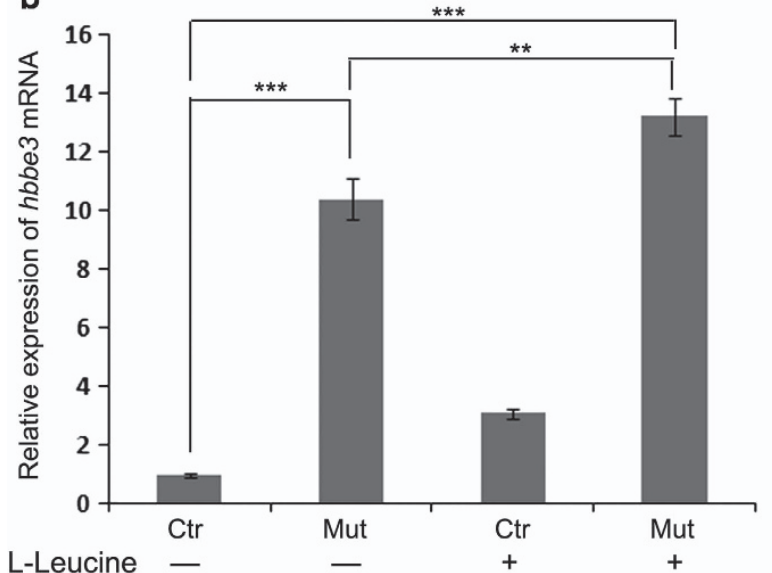

c
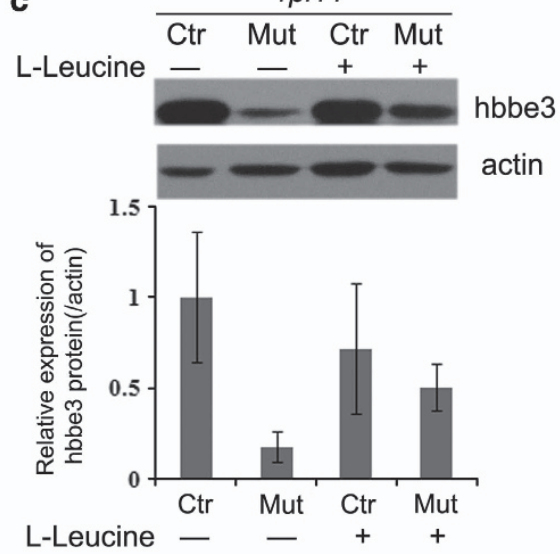

d

L-Leucine

rp/11 Ctr

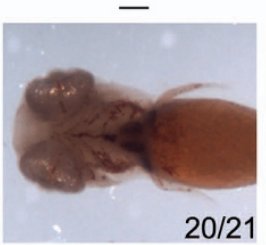

20/21

rpl11 Mut
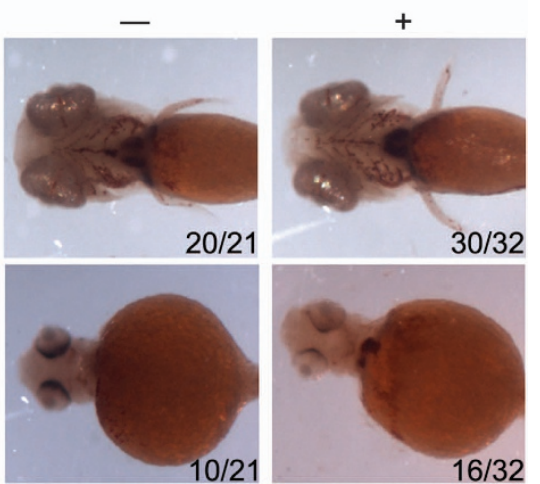

Figure 8 L-Leucine treatment improves the protein production defect of globin gene and partially rescues the anemic phenotype in rp/11 mutant embryos. (a) Similar with rps 19 mutant embryos, rpl11 mutant embryos raised in fish water developed severe edema (black arrow) at $3 \mathrm{dpf}$, while the edema was improved after L-leucine treatment in rp/11 mutant embryos (black arrow). Lateral view with anterior to the left. (b) mRNA expression of $h b b e 3$ was slightly increased in rp/11 mutant embryos after addition of L-leucine compared with mutant embryos in fish water at $3 \mathrm{dpf}$ by real-time PCR analysis. (c) hbbe3 protein expression was detected by western blot analysis. Expression of hbbe3 was dramatically increased after treatment of L-leucine in rp/11 mutant embryos at $3 \mathrm{dpf}$. Histograms represented quantification of western blots. Actin acts as a loading control. (d) Similar with the results of rps 19 mutants, more hemoglobin staining signals were detected in rpl11 mutant embryos treated with L-leucine than the mutant embryos raised in fish water at $4 \mathrm{dpf}$. Ventral view with anterior to the left. The results are representative for two independent experiments. ${ }^{* *} P<0.01 ;{ }^{* \star *} P<0.001 ;$ Student's $t$-test

treated with L-leucine compared to control regardless of rps19 mutation or rp/11 mutation. Meanwhile, protein level of hbbe3 in wild-type and heterozygous siblings was not altered by the addition of L-leucine (Figures 7c and 8c). To address if increasing the protein production of globin gene could lead to the improvement of anemia observed in mutants, we performed o-dianisidine staining for both rps19 and rp/11 mutants. We found that L-leucine treatment partially improved the anemic phenotype in both rps19 and rp/11 mutants (Figures 7d and 8d). Overall, our rps 19 model, as well as rp/11 mutants is responsive to L-leucine treatment. Anemia was improved in both rps 19 and rp/11 mutants with L-leucine treatment, similar to human DBA patients following L-leucine treatment. ${ }^{36}$ Our zebrafish rps19 mutant line appears promising as a useful model towards better understanding the mechanistic defects in DBA and also aids in the discovery of novel therapeutics to treat the disease.

\section{Discussion}

In this study, we generated rps19 mutant zebrafish lines using TALENs. As reported in DBA patients, rps 19 mutants exhibited hematopoietic phenotypes and developed anemia associated with activation of the p53 signaling pathway. We therefore believe that this rps 19 mutant zebrafish should serve as a useful animal model to study the pathogenesis of DBA.

Mechanism of erythroid failure in DBA. RPS19 was the first identified gene whose mutation is currently identified in $25 \%$ of DBA patients. ${ }^{2}$ To date, mutations in at least other nine ribosomal genes, including RPL11, have been reported in DBA patients. ${ }^{12}$ The ribosomal stress and upregulation of the P53 signaling pathway caused by haploinsufficiency of ribosomal proteins are thought to lead to the clinical features of DBA. ${ }^{17,18}$ According to data from in vitro experiments, the specific erythropoietic defects in DBA were thought to be attributed to the hypersensitivity of erythroblasts to the upregulation of TP5 $53^{17}$ and/or the high demand for ribosome synthetic rates of rapidly proliferative erythroid cells. ${ }^{26}$ Other hypotheses, including reduced translation of some specific transcripts essential for erythropoiesis ${ }^{23}$ also exist. Recently, a mutation of GATA1, an erythroid-specific transcription factor, was identified in two siblings and another unrelated DBA patients. ${ }^{37}$ Splicing of GATA1 was impaired by the 
mutation. Mutation of the erythroid-specific gene may contribute to the erythroid-specific phenotype of DBA. However, it remains controversial whether these patients are real DBA cases, because their clinical manifestations did not meet all of the clinical diagnostic criteria for DBA. ${ }^{1}$

For the first time, our in vivo data demonstrated translational defects with globin genes in both rps19 and rp/11 mutants, despite increased or similar transcriptional levels. More importantly, we found that translation was disrupted specifically in erythroid cells in both rps19- and rp/11-deficient zebrafish. This is further supported by observations showing translation of a neutrophil-specific gene was largely unaffected in both mutant zebrafish. We speculate that protein synthesis defects may affect the survival of erythroid cells, for instance, through the activation of cell-cycle arrest and apoptosis in erythroid cells. ${ }^{38}$ These findings will advance our understanding toward the mechanisms of erythroid failure in DBA.

Extra-ribosomal functions of ribosomal proteins. Some ribosomal proteins have been reported to have unexpected functions outside of the ribosome (extra-ribosomal functions). In eukaryotic cells, RPL2, RPL30, RPS14, and RPS28 were reported to function by binding to their own transcripts or some other mechanisms to perform regulation of ribosomal protein production. ${ }^{39-42}$ RPL5, RPL11 and 5S rRNA were found to interact with MDM2, which is responsible for regulating the $p 53$ signaling pathway via the ubiquitination and degradation of $p 53 .{ }^{43-46}$ RPL13a was reported to be involved in a transcript-specific translational control mechanism. RPL13a can be phosphorylated and released from the $60 S$ ribosomal subunit in response to interferon- $\gamma$. Released RPL13a specifically binds the 3'UTR interferon-gammaactivated inhibitor of translation element of its target mRNAs and inhibits translation. ${ }^{47}$

Recently, analysis of RPL38's function provided insight into the specialized ribosome activity controlled by ribosomal proteins. Rp/38 was found to be deleted in tail short (Ts) mice. Rp/38 had higher expression in somites and neural tube, where Ts mice showed tissue-specific phenotype. Interestingly, translation of 8 of the 39 homeobox (Hox) genes, which are essential for the morphological development along the axial skeleton was reduced in Ts mice. The tissue-specific expression pattern of Rp/38 correlated with the specific phenotype observed in Ts mice. ${ }^{48}$ These findings suggested the possibility that increased expression of specific ribosomal proteins in unique tissues might lead to the heterogeneous ribosomes in the distinguished cell tissues by specificities in translating distinct classes of mRNAs. However, this is not the case for rps 19 or rpl11. We checked the expression patterns of $r p s 19, r p / 11$ and $r p / 38$ in zebrafish using RNA whole-mount in situ hybridization. All of them had ubiquitous expression during embryonic development (data not shown). This suggested that the erythroid-specific translational defect exhibited by rps19- and rpl11-deficient zebrafish was not attributed to the tissue-specific expression of $r p s 19$ and $r p / 11$. It is possible that some erythroid-specific ribosome-associated factors, which have interactions with rps 19 and/or rp/11 may mediate this event by inhibiting the translation of erythroid genes. Application of ribosomal profiling strategy will put forward some candidates for this issue.
In summary, utilizing TALENs we generated rps19 mutants in zebrafish. Similar with DBA patients, rps19 mutants had hematopoietic defects and developed anemia; thus, serving as a novel DBA model. Using the newly generated line, we reported the RNA and protein production dissociation of globin genes in rps 19 mutant and confirmed similar findings in rp/11 mutant. Further analysis demonstrates that the dissociation may result from reduced protein production in erythroid cells in rps19- or rp/11-deficient zebrafish. Expression, however, of neutrophil gene was unaffected. For the first time, our in vivo data suggested that the erythroid defects in DBA may be caused by the decreased protein production of erythroid cells in patients. Recently, L-leucine was found to improve the anemia of rps19-deficient zebrafish by activating the mTOR pathway; ${ }^{34}$ however, the underlying mechanism is still under investigation. Our results showed L-leucine treatment alleviated the protein production defects of globin gene and partially rescued the anemic phenotype in both rps 19 and rp/11 mutants. Based on our findings, L-leucine may work through activating translation of erythroid cells. Our findings will aid in the understanding of clinical manifestation of DBA. Additionally, the newly derived rps 19 mutants promise to be useful for investigating the basis for erythroid defects and serve as a tool to carry out therapeutic screens to develop much-needed treatments toward DBA.

\section{Materials and Methods}

Zebrafish lines and maintenance. Zebrafish (Danio rerio) was raised and maintained under standard laboratory conditions at $28.5^{\circ} \mathrm{C}$. All the three constructs for transgenic fish were generated utilizing the Gateway system (Invitrogen, Carlsbad, CA, USA). Approximately, $30 \mathrm{pg}$ of the constructs together with $15 \mathrm{pg}$ Tol2 mRNA were injected into the embryos at one-cell stage; $5 \mathrm{ng}$ of p53 ATG morpholino ${ }^{49}$ was injected into the embryos at one-cell stage for p53 rescue experiment.

Generating rps19 mutant by TALENs. Design of TALENs target site using the online service at: https://tale-nt.cac.cornell.edu/node/add/talen. The sequence-specific TAL effector repeats against the right and left arms (Figure 1a) were constructed using the 'unit assembly' method. ${ }^{27}$ mRNAs encoding left and right TALENs were synthesized in vitro by using the T7 mMessage Machine kit (Ambion, Austin, TX, USA) and purified using a kit from Invitrogen according to the manufactures' instructions. mRNAs were mixed at the ratio of $1: 1$ and co-injected into wild-type zebrafish embryos at one-cell stage at $100 \mathrm{pg} \mathrm{mRNA} / \mathrm{embryo}$ as the final dose. Every three injected and morphologically normal embryos were mixed at $24 \mathrm{hpf}$ to isolate genomic DNA followed by PCR amplification. Total of 24 embryos from each injection group were used to evaluate the efficiency of the generated TALENs. A 305 bp genomic DNA fragment containing the target site was PCR amplified from injected and/or uninjected control embryos. Primers used for this PCR were: forward: $5^{\prime}$-AAGTCCTGGTCTTGGGACAA-3'; reverse: $5^{\prime}-\mathrm{G}$ GAATGGACGAAAGTTACACTG-3'. The PCR products were denatured, annealed and digested with Transgenomic Surveyor Mutation Detection Kit (Transgenomic, Omaha, NE, USA) for Standard Gel Electrophoresis (catalog number: 706020) by following the manufacturer's instruction. The digested PCR products were separated by electrophoresis on $2 \%$ agarose gel and/or $0.8 \%$ PAGE gel.

Real-time PCR. RNA was isolated from 7-15 embryos at $3 \mathrm{dpf}$ using Trizol (Invitrogen) according to the manufacturer's protocol; 2 ug of total RNA was used for cDNA synthesis by reverse transcription using Oligo(dT) ${ }_{12-18}$ (Invitrogen). Realtime PCR was performed using FastStart Universal SYBR Green MasterMix (Roche Diagnostics, Indianapolis, IN, USA) and a MyiQ Single-Color PCR thermal cycler (Biorad, Hercules, CA, USA). Real-time PCR primers used for $p 53$ signaling pathway were from previous literature. ${ }^{19}$ Sequences of real-time PCR primers for hbbe1: RTF: $5^{\prime}$-TTGTGTGGACAGACTTCGAGA-3' and RTR: $5^{\prime}$-TCAGCATAG GTGGCCTTGAT-3'; for hbbe3: RTF: $5^{\prime}$-ATGGTTGTGTGGACAGCTG-3' and RTR: 5'-ATTATCCATGTTGTTGAGAGC-3'; for mpx: RTF3: 5'-GGGGCAGAAGAAGA 
AAGTCC-3' and RTR3: 5'-CCCTTGCTAAACTCTCATCTC-3'; for rps 19: RTF1: 5'-ATGCCAGGTGGTGTAACAGT-3' and RTR1: $5^{\prime}$-GTCATAGAGCCCACGC CAAC-3'; for mCherry. RTF1: $5^{\prime}$-GCGGCACCAACTTCCCCTCC-3' and RTR1: 5'-TCGGCGCGCTCGTACTGTTC-3'; for gata1: RTF: 5'-TGAATGTGTGAAT TGTGGTG-3' and RTR: $5^{\prime}$-ATTGCGTCTCCATAGTGTTG- $3^{\prime} .{ }^{50}$

Whole-mount in situ hybridization. Whole-mount in situ hybridization was conducted as described ${ }^{51}$ using c-myb, mpx, rag $1,{ }^{49} h b b e 1$, and $h b b e 3^{52}$ riboprobes.

Whole-mount o-dianisidine staining. Hemoglobin was measured by whole-mount 0 -dianisidine staining as described previously. ${ }^{49}$

Western blot analysis. Approximately, 15-20 embryos were rinsed and lysed in lysis buffer. Protein concentration was measured with bicinchoninic acid Protein Assay Kit (Thermo Scientific, Rockford, IL, USA) or NanoDrop 1000 Spectrophotometer (Thermo Scientific); $20-40 \mathrm{mg}$ of total protein was employed for western blot analysis. Procedure of western blot was described previously. ${ }^{30}$ Primary antibodies for hbbe1 (AnaSpec, Fremont, CA, USA), mpx (AnaSpec), gata1a (Anaspec), and actin (Sigma, St. Louis, MO, USA) and horseradish peroxidaselinked secondary antibodies (GE Healthcare, Uppsala, Sweden) were used. Synthetic peptide corresponding to the N-terminal region of zebrafish hbbe3 was produced for generating customized primary antibody for hbbe3 (AnaSpec). Western blot results were quantified using ImageJ which was downloaded from NIH website.

Analysis of mCherry fluorescence. For each group, 28-30 embryos were photoed under fluorescent microscope (Zeiss, Oberkochen, Germany) at $3 \mathrm{dpf}$. The fluorescence of mCherry was analyzed using ImageJ. Images were opened using ImageJ software. The same thresholds for both control and mutant embryos were set up to reflect the expression of mCherry throughout the whole embryo. Area, minimum and maximum gray value, integrated density, and mean gray value were measured. Measurements were set as limit to threshold. The whole embryo was measured. The value of area and mean intensity was multiplied to get the intensity of mCherry fluorescence for each embryo.

L-Leucine treatment. Embryos were divided into two groups at $10 \mathrm{hpf}$. One group was raised in fish water with $100 \mathrm{mM}$ L-leucine (Sigma), and the other group was raised in fish water. Embryos were collected for the following experiments at 3 and $4 \mathrm{dpf}$.

Analysis of general translation rate. Overall, $40-80$ embryos at $3 \mathrm{dpf}$ were homogenized and dissociated into single cells with $0.25 \%$ Trypsin-EDTA (Invitrogen). Cells were counted. Comparable amount of cells $\left(\sim 4 \times 10^{5}\right.$ cells) from each group were used. Cells were rinsed twice with methionine-free and cysteine-free DMEM (Invitrogen) followed by incubation in the same medium at $28.5^{\circ} \mathrm{C}$ for one hour. $\mathrm{S}^{35}$-labeled methionine and cysteine (Perkin Elmer, Santa Clara, CA, USA) were added into the culture medium at $10 \mu \mathrm{Ci} / \mathrm{ml}$. After incubation for another $1 \mathrm{~h}$, cells were collected by centrifuging at $4^{\circ} \mathrm{C}$ for $5 \mathrm{~min}$. Cells were rinsed twice with ice-cold phosphate-buffered saline (PBS). The cells were resuspended in $100 \mu$ lysis buffer containing $50 \mathrm{mM}$ Tris- $\mathrm{HCl}(\mathrm{pH} 7.5)$, $150 \mathrm{mM} \mathrm{NaCl}, 1 \%$ Triton X-100, and proteinase inhibitor cocktail (Roche Diagnostics). Proteins in the lysate were precipitated by adding trichloroacetic acid (Sigma-Aldrich) and incubated for $30 \mathrm{~min}$ on ice. Proteins were recovered by vacuum filtration on cellulose filters (Whatman, Uppsala, Sweden) and dried out. The filters were dissolved with scintillation cocktail, and radioactivity from samples were measured with Liquid Scintillation Analyzer (Perkin Elmer). Meanwhile, protein concentration was measured using NanoDrop 1000 Spectrophotometer (Thermo Scientific).

Examination of circulation by angiocardiography. Embryos at $3 \mathrm{dpf}$ were treated with $\times 1$ tricaine. After injecting rhodamine fluorescent dye into their hearts, embryos were immediately observed and pictured with fluorescent microscope (Zeiss); 8-10 embryos for each group were injected and observed.

Analysis of percentage of mCherry-positive cells. Equal number $(30-50)$ of transgenic embryos at $3 \mathrm{dpf}$ were homogenized and dissociated into single cells with $0.25 \%$ Trypsin-EDTA (Invitrogen). Cells were rinsed twice and resuspended in $\times 1 \mathrm{PBS}$. The percentage of mCherry-positive cells was analyzed using BD LSRFortessa cell analyzer (BD Biosciences, San Jose, CA, USA).

\section{Conflict of Interest}

The authors declare no conflict of interest.

Acknowledgements. We thank Dr. Leonard I Zon for providing $\operatorname{Tg}(L C R: G F P)$ zebrafish and plasmids of Gateway elements, members of Lin Lab at the University of California, Los Angeles and Zhang Lab at Peking University and Birong Shen at Peking University for discussion on the manuscript and technical assistance, Jianguo Chen's lab at Peking University for sharing the reagents and assistance in western blot, Dongyi Xu's lab at Peking University for helping with analysis of general translational rate, and Zailing Bai at Peking University for assistance in cell analysis. This work was supported by research grants from NIH (HL097561) to SL and National Natural Science Foundation of China (NSFC; 31110103904) and the 973 program (2012CB945101) to BZ.

\section{Author Contributions}

$Y Z, K M, B Z$, and SL designed the experiments. YZ, ZY, and KM conducted the experiments. $Y Z, B Z$, and $S L$ analyzed the data. $Y Z, J E, B Z$, and $S L$ wrote the paper.

1. Horos R, von Lindern M. Molecular mechanisms of pathology and treatment in Diamond Blackfan Anaemia. Br J Haematol 2012; 159: 514-527.

2. Draptchinskaia N, Gustavsson P, Andersson B, Pettersson M, Willig TN, Dianzani I et al. The gene encoding ribosomal protein S19 is mutated in Diamond-Blackfan anaemia. Nat Genet 1999; 21: 169-175.

3. Willig TN, Gazda H, Sieff CA. Diamond-Blackfan anemia. Curr Opin Hematol 2000; 7: 85-94.

4. Campagnoli MF, Garelli E, Quarello P, Carando A, Varotto S, Nobili B et al. Molecular basis of Diamond-Blackfan anemia: new findings from the Italian registry and a review of the literature. Haematologica 2004; 89: 480-489.

5. Vlachos A, Federman N, Reyes-Haley C, Abramson J, Lipton JM. Hematopoietic stem cell transplantation for Diamond Blackfan anemia: a report from the Diamond Blackfan Anemia Registry. Bone Marrow Transplant 2001; 27: 381-386.

6. Gazda HT, Grabowska A, Merida-Long LB, Latawiec E, Schneider HE, Lipton JM et al. Ribosomal protein $\mathrm{S} 24$ gene is mutated in Diamond-Blackfan anemia. Am J Hum Genet 2006; 79: 1110-1118.

7. Cmejla R, Cmejlova J, Handrkova H, Petrak J, Pospisilova D. Ribosomal protein $\$ 17$ gene (RPS17) is mutated in Diamond-Blackfan anemia. Hum Mutat 2007; 28: 1178-1182.

8. Farrar JE, Vlachos A, Atsidaftos E, Carlson-Donohoe H, Markello TC, Arceci RJ et al. Ribosomal protein gene deletions in Diamond-Blackfan anemia. Blood 2011; 118: 6943-6951.

9. Farrar JE, Nater M, Caywood E, McDevitt MA, Kowalski J, Takemoto CM et al. Abnormalities of the large ribosomal subunit protein, Rpl35a, in Diamond-Blackfan anemia. Blood 2008; 112: 1582-1592.

10. Gazda HT, Sheen MR, Vlachos A, Choesmel V, O'Donohue MF, Schneider $\mathrm{H}$ et al. Ribosomal protein $\mathrm{L} 5$ and $\mathrm{L} 11$ mutations are associated with cleft palate and abnormal thumbs in Diamond-Blackfan anemia patients. Am J Hum Genet 2008; 83: 769-780.

11. Doherty L, Sheen MR, Vlachos A, Choesmel V, O'Donohue MF, Clinton C et al. Ribosomal protein genes RPS10 and RPS26 are commonly mutated in Diamond-Blackfan anemia. Am J Hum Genet 2010; 86: 222-228.

12. Gazda HT, Preti M, Sheen MR, O'Donohue MF, Vlachos A, Davies SM et al. Frameshift mutation in p53 regulator RPL26 is associated with multiple physical abnormalities and a specific pre-ribosomal RNA processing defect in diamond-blackfan anemia. Hum Mutat 2012; 33: 1037-1044.

13. Choesmel V, Bacqueville D, Rouquette J, Noaillac-Depeyre J, Fribourg S, Cretien A et al. Impaired ribosome biogenesis in Diamond-Blackfan anemia. Blood 2007; 109: 1275-1283.

14. Flygare J, Aspesi A, Bailey JC, Miyake K, Caffrey JM, Karlsson S et al. Human RPS19, the gene mutated in Diamond-Blackfan anemia, encodes a ribosomal protein required for the maturation of 40S ribosomal subunits. Blood 2007; 109: 980-986.

15. Idol RA, Robledo S, Du HY, Crimmins DL, Wilson DB, Ladenson JH et al. Cells depleted for RPS19, a protein associated with Diamond Blackfan Anemia, show defects in 18S ribosomal RNA synthesis and small ribosomal subunit production. Blood Cells Mol Dis 2007; 39: 35-43.

16. Robledo S, Idol RA, Crimmins DL, Ladenson JH, Mason PJ, Bessler M. The role of human ribosomal proteins in the maturation of rRNA and ribosome production. RNA 2008; 14: 1918-1929.

17. Dutt S, Narla A, Lin K, Mullally A, Abayasekara N, Megerdichian C et al. Haploinsufficiency for ribosomal protein genes causes selective activation of p53 in human erythroid progenitor cells. Blood 2011; 117: 2567-2576.

18. Jaako P, Flygare J, Olsson K, Quere R, Ehinger M, Henson A et al. Mice with ribosomal protein S19 deficiency develop bone marrow failure and symptoms like patients with Diamond-Blackfan anemia. Blood 2011; 118: 6087-6096. 
19. Danilova N, Sakamoto KM, Lin S. Ribosomal protein S19 deficiency in zebrafish leads to developmental abnormalities and defective erythropoiesis through activation of $\mathrm{p} 53$ protein family. Blood 2008; 112: 5228-5237.

20. Torihara H, Uechi T, Chakraborty A, Shinya M, Sakai N, Kenmochi N. Erythropoiesis failure due to RPS19 deficiency is independent of an activated Tp53 response in a zebrafish model of Diamond-Blackfan anaemia. Br J Haematol 2011; 152: 648-654.

21. Taylor AM, Zon LI. Modeling Diamond Blackfan anemia in the zebrafish. Semin Hemato 2011; 48: 81-88.

22. Cmejlova J, Dolezalova L, Pospisilova D, Petrtylova K, Petrak J, Cmejla R. Translational efficiency in patients with Diamond-Blackfan anemia. Haematologica 2006; 91 : $1456-1464$.

23. Horos R, lispeert H, Pospisilova D, Sendtner R, Andrieu-Soler C, Taskesen E et al. Ribosomal deficiencies in Diamond-Blackfan anemia impair translation of transcripts essential for differentiation of murine and human erythroblasts. Blood 2012; 119: 262-272.

24. Rey MA, Duffy SP, Brown JK, Kennedy JA, Dick JE, Dror Y et al. Enhanced alternative splicing of the FLVCR1 gene in Diamond Blackfan anemia disrupts FLVCR1 expression and function that are critical for erythropoiesis. Haematologica 2008; 93: 1617-1626.

25. Quigley JG, Burns CC, Anderson MM, Lynch ED, Sabo KM, Overbaugh J et al. Cloning of the cellular receptor for feline leukemia virus subgroup C (FeLV-C), a retrovirus that induces red cell aplasia. Blood 2000; 95: 1093-1099.

26. Sieff CA, Yang J, Merida-Long LB, Lodish HF. Pathogenesis of the erythroid failure in Diamond Blackfan anaemia. Br J Haematol 2010; 148: 611-622.

27. Huang $P$, Xiao A, Zhou M, Zhu Z, Lin S, Zhang B. Heritable gene targeting in zebrafish using customized TALENs. Nat Biotechnol 2011; 29: 699-700.

28. Danilova N, Kumagai A, Lin J. p53 upregulation is a frequent response to deficiency of cell-essential genes. PLoS One 2010; 5: e15938.

29. Taylor AM, Humphries JM, White RM, Murphey RD, Burns CE, Zon LI. Hematopoietic defects in rps29 mutant zebrafish depend upon p53 activation. Exp Hematol 2012; 40: 228-237; e225.

30. Danilova N, Sakamoto KM, Lin S. Ribosomal protein L11 mutation in zebrafish leads to haematopoietic and metabolic defects. Br J Haematol 2011; 152: 217-228.

31. Ganis JJ, Hsia N, Trompouki E, de Jong JL, DiBiase A, Lambert JS et al. Zebrafish globin switching occurs in two developmental stages and is controlled by the LCR. Dev Biol 2012; 366: $185-194$

32. Amsterdam A, Nissen RM, Sun Z, Swindell EC, Farrington S, Hopkins N. Identification of 315 genes essential for early zebrafish development. Proc Natl Acad Sci USA 2004; 101 : 12792-12797.

33. Kimball SR, Jefferson LS. New functions for amino acids: effects on gene transcription and translation. Am J Clin Nutr 2006; 83: 500S-507S.

34. Payne EM, Virgilio M, Narla A, Sun H, Levine M, Paw BH et al. L-Leucine improves the anemia and developmental defects associated with Diamond-Blackfan anemia and del(5q) MDS by activating the mTOR pathway. Blood 2012; 120: 2214-2224

35. Jaako P, Debnath S, Olsson K, Bryder D, Flygare J, Karlsson S. Dietary L-leucine improves the anemia in a mouse model for Diamond-Blackfan anemia. Blood 2012; 120: 2225-2228.

36. Pospisilova D, Cmejlova J, Hak J, Adam T, Cmejla R. Successful treatment of a DiamondBlackfan anemia patient with amino acid leucine. Haematologica 2007; 92: e66-e67.

37. Sankaran VG, Ghazvinian R, Do R, Thiru P, Vergilio JA, Beggs AH et al. Exome sequencing identifies GATA1 mutations resulting in Diamond-Blackfan anemia. J Clin Invest 2012; 122: 2439-2443.

38. Gazda HT, Kho AT, Sanoudou D, Zaucha JM, Kohane IS, Sieff CA et al. Defective ribosomal protein gene expression alters transcription, translation, apoptosis, and oncogenic pathways in Diamond-Blackfan anemia. Stem Cells 2006; 24: 2034-2044.
39. Presutti C, Ciafre SA, Bozzoni I. The ribosomal protein L2 in S. cerevisiae controls the level of accumulation of its own mRNA. EMBO J 1991; 10: 2215-2221.

40. Eng FJ, Warner JR. Structural basis for the regulation of splicing of a yeast messenger RNA. Cell 1991; 65: 797-804.

41. Fewell SW, Woolford Jr JL. Ribosomal protein S14 of Saccharomyces cerevisiae regulates its expression by binding to RPS14B pre-mRNA and to 18S rRNA. Mol Cell Biol 1999; 19: 826-834

42. Badis G, Saveanu C, Fromont-Racine M, Jacquier A. Targeted mRNA degradation by deadenylation-independent decapping. Mol Cell 2004; 15: 5-15.

43. Marechal V, Elenbaas B, Piette J, Nicolas JC, Levine AJ. The ribosomal L5 protein is associated with mdm-2 and mdm-2-p53 complexes. Mol Cell Biol 1994; 14: 7414-7420.

44. Lohrum MA, Ludwig RL, Kubbutat MH, Hanlon M, Vousden KH. Regulation of HDM2 activity by the ribosomal protein L11. Cancer Cell 2003; 3: 577-587.

45. Dai MS, Shi D, Jin Y, Sun XX, Zhang Y, Grossman SR et al. Regulation of the MDM2-p53 pathway by ribosomal protein L11 involves a post-ubiquitination mechanism. J Biol Chem 2006; 281: 24304-24313.

46. Zhang Y, Wolf GW, Bhat K, Jin A, Allio T, Burkhart WA et al. Ribosomal protein L11 negatively regulates oncoprotein MDM2 and mediates a p53-dependent ribosomal-stress checkpoint pathway. Mol Cell Biol 2003; 23: 8902-8912.

47. Mazumder B, Sampath P, Seshadri V, Maitra RK, DiCorleto PE, Fox PL. Regulated release of $L 13$ a from the $60 S$ ribosomal subunit as a mechanism of transcript-specific translational control. Cell 2003; 115: 187-198.

48. Kondrashov N, Pusic A, Stumpf CR, Shimizu K, Hsieh AC, Xue S et al. Ribosomemediated specificity in Hox mRNA translation and vertebrate tissue patterning. Cell 2011; 145: 383-397.

49. Zhang Y, Morimoto K, Danilova N, Zhang B, Lin S. Zebrafish models for dyskeratosis congenita reveal critical roles of p53 activation contributing to hematopoietic defects through RNA processing. PLoS One 2012; 7: e30188.

50. Bertrand JY, Kim AD, Teng S, Traver D. CD41 + cmyb + precursors colonize the zebrafish pronephros by a novel migration route to initiate adult hematopoiesis. Development 2008; 135: 1853-1862.

51. Thisse C, Thisse B, Schilling TF, Postlethwait JH. Structure of the zebrafish snail1 gene and its expression in wild-type, spadetail and no tail mutant embryos. Development 1993; 119: $1203-1215$

52. Brownlie A, Hersey C, Oates AC, Paw BH, Falick AM, Witkowska HE et al. Characterization of embryonic globin genes of the zebrafish. Dev Biol 2003; 255 : 48-61

(2) ()ㅇㄱ Cell Death and Disease is an open-access journal published by Nature Publishing Group. This work is licensed under a Creative Commons Attribution-NonCommercialNoDerivs 3.0 Unported License. The images or other third party material in this article are included in the article's Creative Commons license, unless indicated otherwise in the credit line; if the material is not included under the Creative Commons license, users will need to obtain permission from the license holder to reproduce the material. To view a copy of this license, visit http://creativecommons.org/ licenses/by-nc-nd/3.0/ 